\title{
Carbon Monoxide Releasing Molecule-2 Protects Intestinal Mucosal Barrier Function of Rat Undergoing Cardiopulmonary Resuscitation via Attenuation of TNF-a/NF-KB Signaling
}

\author{
Qingsheng Niu \\ Ningxia Medical University \\ Fang Liu \\ Heze Second People's Hospital \\ Jun Zhang \\ General Hospital of Ningxia Medical University \\ Xiaojun Yang \\ General Hospital of Ningxia Medical University \\ Xiaohong Wang ( $\square$ wxhhelen2005@sina.com) \\ General Hospital of Ningxia Medical University
}

\section{Research Article}

Keywords: Carbon monoxide releasing molecule-2, cardiopulmonary resuscitation, intestinal mucosal barrier, TNF-a, NF-kB, tight junctions

Posted Date: July 23rd, 2021

DOl: https://doi.org/10.21203/rs.3.rs-735111/v1

License: @ (i) This work is licensed under a Creative Commons Attribution 4.0 International License. Read Full License 


\section{Abstract}

The unique features of post-cardiac arrest pathophysiology are often superimposed on the disease or injury, causing the cardiac arrest, as well as underlying comorbidities. Exogenous carbon monoxide (CO) was reported to reduce ischemia-reperfusion injury (IRI). This study aimed to assess the effects of CO releasing molecule-2 (CORM-2) on intestinal mucosal barrier function after cardiopulmonary resuscitation (CPR) in rats. For this purpose, we established a rat model of asphyxiation-induced cardiac arrest and resuscitation to study intestinal IRI, and measured the serum level of intestinal fatty-acid binding protein (I-FABP). The expression levels of claudin-3, occludin, ZO-1, tumor necrosis factor-alpha (TNF-a), interleukin-10 (IL-10), and nuclear factor kappa B (NF-kB) p65 were detected by Western blotting. CORM-2 up-regulated the expression levels of tight junction proteins (claudin-3, occludin, and ZO-1) in intestinal mucosa, leading to the reduction of the permeability of intestinal mucosa and reduced the release of proinflammatory cytokines. Besides, the CORM-2 exhibited anti-inflammatory effects by regulating the TNF-a/NF-KB pathway. In conclusion, CORM-2 treatment is clinically significant, preventing intestinal mucosal damage as a result of IRI during CPR.

\section{Introduction}

A systemic ischemia-reperfusion process after cardiopulmonary resuscitation (CPR) can lead to multiple organ dysfunction and post-cardiac arrest syndrome, which is an important cause of high mortality after successful initial resuscitation [1]. Small intestine is extremely susceptible to ischemia-reperfusion injury (IRI) and is exposed to a large number of pathogenic bacteria in the intestinal cavity at the same time [2]. Therefore, the small intestine is a major cause of development of multiple organ failure, due to the release of endotoxins or bacterial translocation. Intestinal tract is vulnerable to IRI, owning to the lack of autonomic mechanisms in the regulation of blood flow [3]. Cold shock is typically mediated by endogenous vasoconstrictors, e.g. noradrenaline, which is considered as a physiological compensatory mechanism in order to provide and stabilize the perfusion of the majority of vital organs, including brain, heart, and lung [4]. Thus, the gut plays a pivotal role in the development of systemic inflammation and multiple organ failure after CPR, therefore further research is needed to prevent gut from IRI during CPR.

Normal intestinal mucosal barrier is composed of mechanical barrier, chemical barrier, immune barrier, and biological barrier, of which the most critical barriers are the mechanical barrier and the immune barrier [5]. Tight junctions (TJs) create a paracellular barrier in epithelial and endothelial cells, protecting them from the external environment, and damaged TJs may lead to the increased intestinal permeability [6]. Enteric bacteria, endotoxins, and macromolecular substances may enter blood through paracellular pathways due to the leaky gut [7]. As $70-80 \%$ of immune cells are located in the gut, translocated bacteria, endotoxins, and other antigens can stimulate the lymphatic tissue in the intestinal mucosa and the body produces an immune response which can initiate the systemic inflammatory response syndrome [8]. Growing evidence indicated that IRI affects mass and function of gut-associated lymphoid tissues (GALTs), and GALTs actively participate in IRI [9]. The damage to the intestinal immune barrier caused by IRI can partly explain the immunosuppression induced by the anti-inflammatory reaction in the later stages of shock. Therefore, it is highly essential to protect the intestinal mucosal barrier function against IRI,which may effectively prevent the onset of multiple organ dysfunction score (MODS) after CPR. However, there is no effective treatment regimen to prevent intestinal barrier dysfunction after CPR.

Carbon monoxide (CO) is traditionally considered as a chemical asphyxiant at high concentrations. However, emerging evidence confirmed that $\mathrm{CO}$ is produced naturally by the human body as a signaling molecule. Endogenous $\mathrm{CO}$ or low concentrations of exogenous $\mathrm{CO}$ have been described to present several cytoprotective functions: anti-apoptosis, anti-inflammatory, vasomodulation, maintenance of homeostasis, stimulation of preconditioning and modulation of cell differentiation [10-11]. COreleasing molecules (CORMs) are being developed as potential therapeutic agents to locally deliver $\mathrm{CO}$ to cells and tissues, thereby overcoming limitations of $\mathrm{CO}$ gas inhalation protocols. Experimental research in animal models has shown the therapeutic potential of CORMs, and the biological effects of CORMs have also been observed in preclinical trials via the genetic modulation of heme oxygenase-1 (HO-1) [12]. However, further research needs to be carried out to indicate whether $\mathrm{CO}$ has a protective effect on the intestinal mucosal barrier against IRI after CPR.

In the present study, a rat model of intestinal IRI after cardiac arrest and cardiopulmonary resuscitation (CA-CPR) was established. We pretreated rats with CORM-2, investigated pathological changes in the colonic mucosa, including the intestinal 
barrier and inflammatory response, and further studied the changes in intestinal TJ proteins in different groups. The aim of this study was to assess the effects of $\mathrm{CO}$ releasing molecule-2 (CORM-2) on intestinal mucosal barrier function in cardiac arrest/CPR rats.

\section{Materials And Methods}

\section{Preparation of animals and establishment of a rat model of intestinal IRI after CA/CPR}

Thirty-two adult healthy male Sprague-Dawley rats (12-16 weeks old, $348 \pm 20 \mathrm{~g}$ ) were purchased from the Experimental Animal Center of Ningxia Medical University (License No. SCXK (Ning) 2015-0001). All of the animal studies were performed in accordance with the Guidelines for the Care and Use of Laboratory Animals of the National Institutes of Health and were approved by the Institutional Animal Care and Use Committee of Ningxia Medical University (NO. 2019-022). Rats were kept in the Animal Center of the Brain Laboratory of Ningxia Medical University at a uniform temperature of $20-22^{\circ} \mathrm{C}$ and under a light/dark cycle of $12 \mathrm{~h}$. After fasting overnight, with the exception of free access to water, the rats were anesthetized by the intraperitoneal injection of $2 \%$ sodium pentobarbital $(45 \mathrm{mg} / \mathrm{kg}$ ), and then, were stretched supinely on a surgical board. The rats were mechanically air-ventilated with a tidal volume of $20 \mathrm{ml} / \mathrm{kg}$ in a manner with a rate of $100 \mathrm{breaths} / \mathrm{min}$. The left femoral artery was cannulated with polyethylene (PE) 50 catheters into the aorta for monitoring arterial pressure. A PE-50 catheter was advanced from the right carotid artery into the left ventricle (LV) for hemodynamic monitoring. The right external jugular vein was cannulated by a $4 \mathrm{~F} \mathrm{PE}$ catheter and advanced into the right atrium for injection of $4 \%$ potassium chloride and $10 \%$ epinephrine. Surface electrocardiogram (ECG) was performed with electrodes placed on the skin. When a rat was under general anesthesia, $4 \%$ potassium chloride $(0.12 \mathrm{ml} / 100 \mathrm{~g})$ was rapidly injected through the right jugular vein, and the tracheal intubation was clamped at the same time, resulting in cardiac arrest (mean arterial pressure (MAP) $\leq 20 \mathrm{mmHg}$ ) lasting for 4 min. After cardiac arrest, CPR was started that lasted for 3 min with the chest compression at a rate of 100/min and mechanical ventilation with delivering tidal volumes of $20 \mathrm{ml} / \mathrm{kg}$. The compression-to-ventilation ratio was $5: 4$. At the beginning of CPR, 10\% epinephrine $(0.04 \mathrm{mg} / \mathrm{kg})$ was administrated through the right jugular vein. Return of spontaneous circulation (ROSC) was defined as return of a supraventricular rhythm with a MAP $\geq 60 \mathrm{mmHg}$ lasting for $\geq 5 \mathrm{~min}$. Besides, the hemodynamics was monitored continuously for $4 \mathrm{~h}$ after successful resuscitation. Failed CPR was declared if animals had no ROSC after 5 min of CPR.

\section{Reagents}

Tricarbonyldichlororuthenium (II) dimer ((Ru(CO)3Cl2)2 or CORM-2) was purchased from Sigma-Aldrich (St. Louis, MO, USA), and $1 \mathrm{mg} / \mathrm{ml}$ CORM-2 solution was made freshly before use by dissolving CORM-2 powder into normal saline containing $2 \%$ dimethyl sulfoxide (DMSO). The inactivate CORM-2 (iCORM-2) solution was prepared by adding CORM-2 into DMSO and leaving the mixture in a $5 \%$ carbon dioxide-containing humidified atmosphere at $37^{\circ} \mathrm{C}$ for $24 \mathrm{~h}$ to release the $\mathrm{CO}$.

\section{Experimental Procedure}

Rats were randomly assigned into the following 4 groups ( $n=8$ in each group): (1) sham-operated group (rats were instrumented with catheter without undergoing CA/CPR, and were injected intraperitoneally with an equal volume of normal saline, (2) CPR group (aortic catheters were placed into adult rats and underwent CA/CPR), (3) CORM-2 group and (4) iCORM-2 group. The rats in the three groups all received CA/CPR. In the CORM-2 group, the rats were administrated CORM-2 (4 mg/kg, dissolved in $2 \%$ DMSO and diluted in normal saline) by the intraperitoneal injection at $12 \mathrm{~h}$ before commencing the CA/CPR. In the iCORM-2 group, the rats were administrated iCORM-2 (4 mg/kg, diluted in the same condition as CORM-2) at the same time point. In the CPR groups, animals were separately administrated $0.9 \%$ saline at the same time point. The hemodynamic data were monitored for $4 \mathrm{~h}$ after $\mathrm{CA} / \mathrm{CPR}$ (or catheterization) in each group. For each rat, the last $5 \mathrm{~cm}$ of the small intestine (ileum) was excised and blood samples of the rats were harvested at $4 \mathrm{~h}$ after operation.

\section{Determination of serum concentration of intestinal fatty-acid binding protein (I-FABP)}


To detect the serum concentration of I-FABP, $100 \mu \mathrm{l}$ of standard or sample was added into each well of the microplate and incubated for $90 \mathrm{~min}$ at $37^{\circ} \mathrm{C}$. After discarding the liquid in the well, $100 \mathrm{ul}$ of biotinylated working solution (Elabscience Biotechnology, Wuhan, China) was added into each well and incubated for $1 \mathrm{~h}$ at $37^{\circ} \mathrm{C}$. Decant the first primary antibody solution and wash the wells for three times with phosphate-buffered saline (PBS), add $100 \mu$ l of horseradish peroxidase (HRP) solution (Elabscience Biotechnology) into each well, and incubate for $30 \mathrm{~min}$ at $37^{\circ} \mathrm{C}$. Finally, the substrate solution and the stop solution were added into the sample, and the optical density value was immediately measured at the wavelength of $450 \mathrm{~nm}$.

\section{Histopathologic evaluation}

lleum was excised after $4 \mathrm{~h}$ of ROSC. The ileum tissue was fixed in $4 \%$ paraformaldehyde, partially embedded into paraffin, cut into 6-mm thick sections, and stained with hematoxylin and eosin. Each ileum section was analyzed with an optical microscope by a researcher who was blinded to experimental conditions. The morphological integrity of the intestinal wall was classified using a modified protocol presented by the Chiu et al.: Grade 0: normal mucosa; Grade 1: development of a sub-epithelial space at the tips of the villi; Grade 2: more extended sub-epithelial space at the tips of the villi, development of Gruenhagen's space at the tips of the villi; Grade 3: massive epithelial lifting down the sides of the villi, villus necrosis; Grade 4: villi are denuded of epithelial layer; Grade 5: loss of villi, mucosal ulceration and necrosis with invasion of the muscularis propria [13].

\section{Transmission electron microscopy}

The ileum tissues were harvested and cut into $1-\mathrm{mm}^{3}$ slices, fixed in $3 \%$ glutaraldehyde for $2 \mathrm{~h}$ at $4{ }^{\circ} \mathrm{C}$, post-fixed in $1 \%$ osmium tetroxide, dehydrated, and embedded into epoxy resin. According to the standard principle of three-dimensional localization, 50 $\mathrm{nm}$ tissue sections were randomly cut, mounted on copper mesh, double stained with lead citrate and uranyl acetate, and were then observed by a transmission electron microscope.

\section{Detection of differential expressions of TJ proteins}

Protein was extracted from ileum tissue of each rat and placed in lysis buffer to detect the expression levels of claudin-3, ZO-1, and occludin. Protein samples (30 $\mathrm{\mu g}$ /each sample) were separated by $10 \%$ sodium dodecyl sulfate (SDS)-polyacrylamide gel and transferred onto polyvinylidene difluoride (PVDF) membranes. After blocking the nonspecific binding sites with $5 \%$ nonfat milk for $60 \mathrm{~min}$, the membranes were incubated overnight at $4{ }^{\circ} \mathrm{C}$ with the primary antibodies, including ZO-1/TJP1 (dilution, 1:500; Thermo Fisher Scientific, Waltham, MA, USA), occludin (dilution, 1:200; Thermo Fisher Scientific), and anti-claudin-3 (dilution, 1:200; Abcam, Cambridge, UK). The membranes were incubated with horseradish peroxidase (HRP)-conjugated secondary antibody (Cell Signaling Technology, Danvers, MA, USA) for $60 \mathrm{~min}$ at room temperature. The binding was visualized using the ECL (enhanced chemiluminescence) Plus reagents (Cell Signaling Technology). The relative protein expression levels were quantified by the ImageJ software (National Institutes of Health, Bethesda, MD, USA). All experiments were performed in triplicate.

\section{Cytokine assays}

The ileum tissues were harvested and homogenized in PBS. The concentrations of cytokines in the ileum tissues were detected by Western blotting. Anti-tumor necrosis factor-alpha (TNF-a), anti-nuclear factor kappa B (NF-KB) p65, and anti-interleukin-10 (IL10) antibodies were obtained from Abcam. The images were captured and analyzed by the KODAK Image Station $4000 R$ (IS4000R) system.

\section{Statistical analysis}

Statistical analysis was performed by the SPSS 22.0 software (IBM, Armonk, NY, USA). The data were presented as mean \pm standard deviation (SD). Normally distributed data were analyzed by the Shapiro-Wilk test or Fisher's exact test, as appropriate. Outcomes were compared among 4 groups by one-way analysis of variance (ANOVA) post hoc tests, Outcome change (over time) of intra-group was performed by Repeated Measure ANOVA. P-value $<0.05$ was considered statistically significant.

\section{Results}

\section{Hemodynamic data}


In the present study, hemodynamic changes of rats were monitored at various time points (Table 1). No significant difference was found in the MAP of baseline in the sham-operated group and CPR group. In the CPR, CORM-2, and iCORM-2 groups, the MAP at each time point after resuscitation was markedly lower than that of baseline and this phenomenon was observed until 4 $\mathrm{h}$ after resuscitation $(\mathrm{P}<0.05$; Table 1$)$. The MAP at each time point after resuscitation in all the resuscitated-based groups was significantly lower than that at the same time point in the sham-operated group $(P<0.05$; Table 1$)$. The MAP at each time point after resuscitation in the CORM-2 group was noticeably higher than that in the CPR and iCORM-2 groups $(P<0.05 ;$ Table 1$)$.

\section{Effects of CORM-2 on the alleviation of damage to ileum tissues in rats after CPR}

Figure 1 shows the histological changes in the intestinal tissues of rats in each group. Rats in the sham-operated group exhibited an intact ileal mucosa, neat intestinal villi, deep crypts, and a clear and complete gland structure. In the ileum of rats in the CPR group, villi arranged in a disorderly fashion, mucosal swelling, villi edema, partial villi destruction, and extensive necrosis in the intestinal epithelial cells. CORM-2 group showed a mild mucosal edema, a small amount of necrosis in the intestinal epithelial cells, and partial villi destruction. CORM-2, rather than iCORM-2, reduced intestinal epithelial damage, which improved morphological changes in the intestinal mucosa during CA-CPR. Compared with the sham-operated group, Chiu's scores for intestinal mucosal injury in all resuscitation-based groups were elevated $(4.59 \pm 0.34,2.38 \pm 0.38$, and $3.64 \pm 0.53$ vs. $0.40 \pm 0.13$; $\mathrm{P}<0.05)$, and the Chiu scores in the CORM-2 group were significantly lower than those in the CPR and iCORM-2 groups $(2.38 \pm 0.38$ vs. $4.59 \pm 0.34$ and $3.64 \pm 0.53, P<0.05)$.

TEM revealed ultrastructural changes in the intestinal mucosal tissues in all the groups. As illustrated in Figure 3 , in the shamoperated group, the epithelial cells were closely arranged, and microvilli on the surface of epithelial cells were arranged in neat rows. The TJs and desmosomes were clear and complete, and the paracellular spaces were narrow. In the CPR group, the microvilli were sparse with an irregular length and arrangement (Figure 2B). The TJs and desmosomes were obscured or disappeared, mitochondria were swollen and vacuolated, and the paracellular spaces were wider. Compared with the CPR and iCORM-2 groups, CORM-2 improved ultrastructural integrity of intestinal mucosa in the CA-CPR group. However, no significant improvement by iCORM-2 treatment was detected.

\section{Serum I-FABP level}

I-FABP serves as a biomarker of enterocyte damage. The results showed that compared to the sham-operated group, the I-FABP level was significantly elevated in the CPR and iCORM-2 groups (585.64 \pm 119.84 and $414.12 \pm 36.13$ vs. $230.19 \pm 37.01, P<0.05$, Table 2). However, the CORM-2 treatment noticeably decreased the serum I-FABP level in the CORM-2 group (306.10 \pm 19.22 vs. $585.64 \pm 119.84, P<0.05)$. Taken together, the data suggested that the CORM-2 treatment could reduce intestinal IRI in the CA-CPR group.

\section{Effects of CORM-2 on the expression levels of claudin-3, occludin, and ZO-1 in ileum tissue}

To study the mechanisms underlying the CO-mediated alleviation of the increased intestinal permeability in the CA/CPR group, the expression levels of claudin-3, ZO-1, and occluding, as markers of intestinal barrier function and permeability, were detected by Western blotting. Figure 3 displays that the expression levels of claudin-3, occludin, and ZO- 1 in the intestinal mucosa were reduced in the CPR, CORM-2, and iCORM-2 groups $(P<0.05)$ compared with those in the sham-operated group. However, the expression levels of occludin, claudin-3, and ZO-1 $(P<0.05)$ were markedly elevated in the CORM-2 group compared with those in rats that received CPR or those underwent CPR + iCORM-2. The above-mentioned findings showed that CORM-2, rather than iCORM-2, increased the levels of TJ proteins in the CPR group.

\section{Effects of CORM-2 on the expression levels of TNF- $a$, IL-10, and NF-KB p65 in ileum tissue}

To indicate whether $\mathrm{CO}$ can inhibit the intestinal inflammation in rats that received CA/CPR, the expression levels of TNF- $a$, IL-10, and NF-KB p65 were detected by Western blotting. The expression levels of TNF-a and IL-10 in the CPR and iCORM-2 groups were significantly elevated compared with those in the sham-operated group $(P<0.05$, Figure 4). The expression level of TNF-a decreased in the CORM-2 group compared with that in the CPR group $(P<0.05)$, and the expression level of IL-10 increased compared with that in the sham-operated group, while no significant difference was detected $(P>0.05)$. Compared with the sham- 
operated group, the expression level of NF-KB p65 was significantly elevated in the CPR and iCORM-2 groups $(P<0.05)$, However, the expression level of NF-KB in the CORM-2 group did not significantly change $(P>0.05)$. Collectively, $C O$ plays an antiinflammatory role in intestinal injury. The anti-inflammatory role of $\mathrm{CO}$ is associated with inhibiting the activation of NF-KB and reducing the release of the pro-inflammatory factors.

\section{Discussion}

As defined by the American Heart Association and the American College of Cardiology, "(sudden) cardiac arrest is the sudden cessation of cardiac activity, so that a victim becomes unresponsive, with no normal breathing and no signs of circulation [14]. CPR is an emergency procedure that combines chest compressions often with artificial ventilation in an effort to manually preserve intact brain function until further measures are taken to restore spontaneous blood circulation and breathing in a person who is in cardiac arrest [15-16]. The intestine is the organ where ischemia occurs first and blood perfusion recovery is mainly delayed during CPR [17]. Intestinal ischemia can induce a systemic inflammatory response and gut-origin infection related to bacterial translocation that may cause multiple organ failure after resuscitation [8]. In the present study, we demonstrated that CORM-2 possessed protective effects against post-resuscitation IRI by attenuating inflammation response and up-regulating the expression levels of intestinal TJ proteins. Additionally, we found that the MAP of rats treated with CORM-2 at each time point after CPR was higher than that in other CPR-based groups. Therefore, our results suggested that pre-treatment with CORM-2 by intraperitoneal injection at $12 \mathrm{~h}$ before CPR can stabilize hemodynamics. CORMs have been reported to possess a vasodilatory effect that involves the activation of soluble guanylyl cyclase and the large-conductance Ca2+-activated $\mathrm{K}+\mathrm{channel}$ (BKCa channel) $[10,18]$. However, the changes of MAP due to CORM in rats undergoing CPR after ROSC were discordant with the vasodilatory effect of CORM. The increased MAP in rats that were treated with CORM-2 could be attributed to two factors. First, according to our previous study, CORM-2 increased cardiac output and left ventricular ejection fraction and exerted a positive effect on ischemic myocardial mitochondrial function and ultrastructural changes to protect cardiac tissues of lipopolysaccharide (LPS)-stimulated rats [19]. This beneficial effect on cardiac function by CORM-2 treatment might result in the increase of MAP in rats. Second, the protective effect of CORM-2 on the vascular endothelium during IRI might partly explain the elevation of MAP in CA/CPR rats, because healthy blood vessels are maintained by moderate levels of CO via crosstalk with eNOS/NO pathway [20-21]. Hence, the administration of CORM-2 during CA-CPR exerts a therapeutic effect against hemodynamic deterioration induced by the cardiac arrest.

To our knowledge, the intestinal villi are extremely susceptible to ischemic damage and their necrosis is one of the earliest histological changes that occur during intestinal ischemia. The results of the current study confirmed that CORM-2 attenuated IRI in a rat model of CA/CPR. We found that I-FABP, as a marker for intestinal damage, was localized in epithelial cells of the small intestine, and the serum I-FABP level decreased in CORM-2 group compared with that in the CPR group. Meanwhile, pathological changes of intestinal mucosa observed by light microscopy showed that CORM-2 treatment mitigated villi edema, goblet cell swelling, and inflammatory cell infiltration, and reduced Chiu's score. Additionally, intraperitoneal injection of CORM-2 resulted in protection against intestinal IRI in rats. The TEM observations indicated that $\mathrm{CO}$ could preserve microvilli on the surface of epithelial cells. Notably, the key role of CORM-2 in protecting intestinal mechanical barrier is the maintenance of paracellular spaces and TJs, playing a significant role in selective paracellular permeability [22].

As we confirmed that CORM-2 could maintain intestinal epithelial barrier integrity, we used Western blotting to detect the expression levels of claudin-3, ZO-1, and occludin in ileum tissues. The result showed that pre-treatment with CORM-2 significantly up-regulated the expression levels of claudin-3, ZO-1, and occludin, and CORM-2 could promote the transcription and translation of the TJ proteins in rats undergoing CA/CPR. Our findings confirmed that the protective effect of CORM-2 against $\mathrm{CA} / \mathrm{CPR}$-induced intestinal permeability is associated with the modulation of the expressions of TJ proteins. Moreover, no significant change was detected in the expression level of occludin between the sham-operated group and CORM-2 group, which is highly expressed at cell-cell contact sites and is important in the assembly and maintenance of TJ proteins [23]. Consequently, CORM-2 plays a noticeable role in reducing intestinal TJ injury induced by ischemia reperfusion in rats undergoing CA/CPR.

The mechanism of $\mathrm{CO}$ regulating assembly of TJ-related proteins after undergoing CA/CPR has still remained elusive. In recent years, growing evidence suggested that intestinal mucosal damage may be triggered by the release of pro-inflammatory

Page 6/14 
cytokines via activation of gut mucosal immune system [24-25]. TNF-a may play a key role in the chain reaction of proinflammatory factors, triggering TJ structural damage. The protective effect realizing the maintenance of TJ proteins is exerted via down-regulation of myosin light chain kinase (MLCK) and phosphorylated myosin light chain. The MLCK is a kinase that leads to fast/transitory changes in paracellular permeability by phosphorylation of myosin II regulatory light chain [26]. Activation of MAPKs and NF- $\kappa$ B pathways causes proinflammatory cytokines, such as interleukin- 6 (IL-6), IL-8, and TNF- $a$ to be released, which may lead to the inflammatory response [27]. The present research revealed that IRI may increase the expression levels of TNF- $a$ and NF-KB, which is consistent with findings of previous studies. However, the administration of CORM-2 reduced the expression levels of TNF- $\alpha$ and NF-KB in ileum tissue. Therefore, it can be concluded that CORM-2 can alleviate downregulation of the expressions of TJ proteins induced by IRI in rats undergoing CA/CPR by reducing the TNF-a expression via the NF-KB signaling pathway. However, the protective effects of CORM-2 on small intestinal tight junctions can be justified by stabling hemodynamic changes to alleviate IRI [28-29]. In addition, our results showed that the inactive CORM-2 only had a slight effect on inhibiting NF-KB, while it had no influence on TNF-a in rats undergoing CPR, because the molecule of CORM-2 has a long-lasting CO-releasing monomer form, and it is almost difficult to completely inactivate CORM-2 in DMSO [30].

The results of the current research demonstrated that CORM-2 exerts anti-inflammatory effects by regulating the TNF-a/NF-KB pathway. To the best of our knowledge, anti-inflammatory properties of $\mathrm{CO}$ are not only associated with the inhibition of proinflammatory cytokines, but also with activation of anti-inflammatory cytokines. IL-10 is a pleiotropic cytokine that controls inflammatory processes by suppressing the production of proinflammatory cytokines [31]. The results of the present research revealed that CORM-2 reduced the expression level of IL-10 in ileum tissue, and this result is inconsistent with the previously reported finding, in which treatment with CORM could increase the expression level of IL-10 in rats with severe autoimmune diseases [32-33]. However, our results are in agreement with a recent study demonstrated that pretreatment with CORM-2 could significantly decrease the serum concentrations of transforming growth factor beta-3 (TGF- $\beta 3$ ), IL-10, TNF-a, interferon gamma (IFN-y), and granulocyte-macrophage colony stimulating factor (GM-CSF) in rats subjected to IRI compared with vehicle control group [34]. Although we did not indicate how CORM-2 could down-regulate the expression level of IL-10, we found that the antiinflammatory properties of $\mathrm{CO}$ could not be mediated by IL-10, partially through the directly inhibition of the expression level of NF-KB after CPR. Taken together, the mechanism underlying CO-induced decrease of IL-10 in intestinal IRI requires further researches.

In summary, $\mathrm{CO}$ showed to play a protective role in intestinal mucosal barrier in rats undergoing CPR. CORM-2 up-regulated the expression levels of TJ proteins (e.g., claudin-3, occludin, and ZO-1) in intestinal mucosa, leading to the reduction of the permeability of intestinal mucosa. Besides, the CORM-2 exhibited anti-inflammatory effects by regulating the TNF-a/NF-KB pathway. Overall, CORM-2 treatment is clinically significant, preventing intestinal mucosal damage as a result of IRI during CPR.

\section{Declarations}

\section{AUTHOR CONTRIBUTION}

Qingsheng Niu: Data curation, Writing - original draft, Writing - review \& editing, writing manuscripts. Fang Liu: Experimental operation, data statistics. Jun Zhang: Writing - original draft, experimental technical guidance. Xiaojun Yang: Auxiliary experiment operation. Xiaohong Wang: Writing - review \& editing, Experimental design, Supervision, review, and revision of manuscripts.

\section{FUNDING}

This work was supported by the National Natural Science Foundation of China (No. 81460288 and No.81960348)

\section{AVAILABILITY OF DATA AND MATERIAL}

The data used to support the findings of this study are available from the corresponding author upon request.

Ethics Approval and Consent to Participate. Approval was obtained from the Institutional Animal Care and Use Committee of Ningxia Medical University. The procedures used in this study adhere to the Care and Use of Laboratory Animals of the National Institutes of Health. 
Consent to participate: Not applicable.

Consent for Publication: Not applicable.

Conflict of Interest: The authors declare no competing interests.

\section{References}

1. Adrie C, Adib-Conquy M, Laurent I, et al. Successful cardiopulmonary resuscitation after cardiac arrest as a "sepsis-like" syndrome (J). Circulation, 2002, 106(5):562-568.

2. ArRajab A, Dawidson I, Fabia R. Reperfusion injury (J). New Horiz, 1996, 4(2):224-234.

3. Davidson MT, Deitch EA, Lu Q, et al. Trauma-hemorrhagic shock mesenteric lymph induces endothelial apoptosis that involves both caspase-dependent and caspase-independent mechanisms (J). Ann Surg, 2004, 240(I):123-131.

4. Nilsen JH, Valkov S, Mohyuddin R, Schanche T, Kondratiev TV, Naesheim T, Sieck GC, Tveita T. Study of the Effects of $3 \mathrm{~h}$ of Continuous Cardiopulmonary Resuscitation at $27^{\circ} \mathrm{C}$ on Global Oxygen Transport and Organ Blood Flow. Front Physiol. 2020 Apr 16;11:213.

5. Schellekens DH,Grootjans J,Dello SA,et al. Plasma intestinal fatty acid-binding protein levels correlate with morphologic epithelial intestinal damage in a human translational ischemia-reperfusion model(J).J Clin Gastroenterol,2014,48(3):253260.

6. Lee B, Moon KM, Kim CY. Tight Junction in the Intestinal Epithelium: Its Association with Diseases and Regulation by Phytochemicals. J Immunol Res. 2018 Dec 16;2018:2645465.

7. Mennini FS, Russo S, Marcellusi A, et al. Economic effects of treatment of chronic kidney disease with low-protein $\operatorname{diet}(\mathrm{J})$. J Ren Nutr,2014,24:313-321.

8. Vighi G, Marcucci F, Sensi L, Di Cara G, Frati F. Allergy and the gastrointestinal system. Clin Exp Immunol. 2008 Sep;153 Suppl 1(Suppl 1):3-6.

9. Nagase M, Sakurai A, Sugita A, et al. Oxidative stress and abnormal cholesterol metabolism in patients with post-cardiac arrest syndrome(J). J Clin Biochem Nutr, 2017,61:108-117.

10. Rotko D, Bednarczyk P, Koprowski P, Kunz WS, Szewczyk A, Kulawiak B. Heme is required for carbon monoxide activation of mitochondrial BKCa channel. Eur J Pharmacol. 2020 Aug 15;881:173191.

11. Otterbein LE, Foresti R, Motterlini R. Heme Oxygenase-1 and Carbon Monoxide in the Heart: The Balancing Act Between Danger Signaling and Pro-Survival. Circ Res. 2016 Jun 10;118(12):1940-1959.

12. Ling K, Men F, Wang WC, Zhou YQ, Zhang HW, Ye DW. Carbon Monoxide and Its Controlled Release: Therapeutic Application, Detection, and Development of Carbon Monoxide Releasing Molecules (CORMs). J Med Chem. 2018 Apr 12;61(7):26112635.

13. Chiu CJ, McArdle AH, Brown R, Scott HJ, Gurd FN. Intestinal mucosal lesion in low-flow states. I. A morphological, hemodynamic, and metabolic reappraisal. Arch Surg. 1970 Oct;101(4):478-83.

14. Chugh SS, Reinier K, Teodorescu C, Evanado A, Kehr E, Al Samara M, Mariani R, Gunson K, Jui J. Epidemiology of sudden cardiac death: clinical and research implications. Prog Cardiovasc Dis. 2008 Nov-Dec;51(3):213-28.

15. Jiang L, Zhang JS. Mechanical cardiopulmonary resuscitation for patients with cardiac arrest. World J Emerg Med. 2011;2(3):165-8.

16. Lurie KG, Nemergut EC, Yannopoulos D, Sweeney M. The Physiology of Cardiopulmonary Resuscitation. Anesth Analg. 2016 Mar;122(3):767-83.

17. Penn AH, Schmid-Schönbein GW. Severe intestinal ischemia can trigger cardiovascular collapse and sudden death via a parasympathetic mechanism. Shock. 2011 Sep;36(3):251-62.

18. Siow RC, Sato H, Mann GE. Heme oxygenase-carbon monoxide signalling pathway in atherosclerosis: anti-atherogenic actions of bilirubin and carbon monoxide? Cardiovasc Res. 1999 Feb;41(2):385-94. 
19. Zhang S, Xu Y, Zhu J, Ma J, Niu Q, Wang X. Carbon monoxide attenuates LPS-induced myocardial dysfunction in rats by regulating the mitochondrial dynamic equilibrium. Eur J Pharmacol. 2020 Dec 15;889:173726.

20. Choi YK, Kim YM. Regulation of Endothelial and Vascular Functions by Carbon Monoxide via Crosstalk With Nitric Oxide. Front Cardiovasc Med. 2021 Apr 12;8:649630.

21. Ozaki KS, Kimura S, Murase N. Use of carbon monoxide in minimizing ischemia/reperfusion injury in transplantation. Transplant Rev (Orlando). 2012 Apr;26(2):125-39.

22. Günzel D, Yu AS. Claudins and the modulation of tight junction permeability. Physiol Rev. 2013 Apr;93(2):525-69.

23. Lee B, Moon KM, Kim CY. Tight Junction in the Intestinal Epithelium: Its Association with Diseases and Regulation by Phytochemicals. J Immunol Res. 2018 Dec 16;2018:2645465.

24. Yang $X, B a i H$, Wang $Y$, et al.Deletion of regulatory $T$ cells supports the development of intestinal ischemia-reperfusion injuries(J). J Surg Res,2013,184(2):832-837.

25. Geha M, Tsokos MG, Bosse RE, et al.IL-17A Produced by Innate Lymphoid Cells Is Essential for Intestinal IschemiaReperfusion Injury(J). J Immunol,2017,199(8):2921-2929.

26. Cunningham KE, Turner JR. Myosin light chain kinase: pulling the strings of epithelial tight junction function. Ann N Y Acad Sci. 2012 Jul;1258(1):34-42.

27. Wu Y, Zhou BP. TNF-alpha/NF-kappaB/Snail pathway in cancer cell migration and invasion. Br J Cancer. 2010 Feb 16;102(4):639-44.

28. Andersson EA, Mallard C, Ek CJ. Circulating tight-junction proteins are potential biomarkers for blood-brain barrier function in a model of neonatal hypoxic/ischemic brain injury. Fluids Barriers CNS. 2021 Feb 10;18(1):7.

29. Wu TK, Lim PS, Jin JS, Wu MY, Chen CH. Impaired Gut Epithelial Tight Junction Expression in Hemodialysis Patients Complicated with Intradialytic Hypotension. Biomed Res Int. 2018 Jan 16;2018:2670312.

30. Motterlini R, Clark JE, Foresti R, Sarathchandra P, Mann BE, Green CJ. Carbon monoxide-releasing molecules: characterization of biochemical and vascular activities. Circ Res. 2002 Feb 8;90(2):E17-24.

31. Driessler F, Venstrom K, Sabat R, Asadullah K, Schottelius AJ. Molecular mechanisms of interleukin-10-mediated inhibition of NF-kappaB activity: a role for p50. Clin Exp Immunol. 2004 Jan;135(1):64-73.

32. Chen P, Sun B, Chen H, Wang G, Pan S, Kong R, Bai X, Wang S. Effects of carbon monoxide releasing molecule-liberated CO on severe acute pancreatitis in rats. Cytokine. 2010 Jan;49(1):15-23.

33. Uddin MJ, Li CS, Joe Y, Chen Y, Zhang Q, Ryter SW, Chung HT. Carbon Monoxide Inhibits Tenascin-C Mediated Inflammation via IL-10 Expression in a Septic Mouse Model. Mediators Inflamm. 2015;2015:613249.

34. Magierowska K, Korbut E, Hubalewska-Mazgaj M, Surmiak M, Chmura A, Bakalarz D, Buszewicz G, Wójcik D, Śliwowski Z, Ginter G, Gromowski T, Kwiecień S, Brzozowski T, Magierowski M. Oxidative gastric mucosal damage induced by ischemia/reperfusion and the mechanisms of its prevention by carbon monoxide-releasing tricarbonyldichlororuthenium (II) dimer. Free Radic Biol Med. 2019 Dec;145:198-208

\section{Tables}

Table 1 Hemodynamic Data Before and After Resuscitation 


\begin{tabular}{|c|c|c|c|c|c|c|c|}
\hline Item & Time point & $\begin{array}{l}\text { Sham- } \\
\text { operated } \\
\text { group } \llbracket n=8 \rrbracket\end{array}$ & $\begin{array}{l}\text { CPR group } \\
\bigotimes n=8 \rrbracket\end{array}$ & 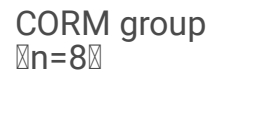 & 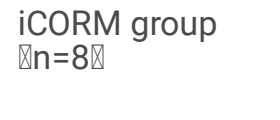 & $\begin{array}{l}\text { F- } \\
\text { value }\end{array}$ & $\begin{array}{l}\mathrm{P} \text { - } \\
\text { value }\end{array}$ \\
\hline \multirow{6}{*}{$\begin{array}{l}\text { MAP } \\
\rrbracket \mathrm{mmHg} \rrbracket\end{array}$} & Baseline & $136.21 \pm 6.22$ & $133.03 \pm 4.68$ & $135.49 \pm 4.8$ & $135.57 \pm 4.79$ & 0.588 & 0.628 \\
\hline & $\begin{array}{l}0.5 \mathrm{~h} \text { after } \\
\text { resuscitation (or } \\
\text { catheterization) }\end{array}$ & $136.46 \pm 5.73$ & $93.01 \pm 5.15 \Delta a$ & $110.08 \pm 3.84 \Delta a b$ & $96.80 \pm 3.79 \Delta \mathrm{ac}$ & 139.983 & 0.000 \\
\hline & $\begin{array}{l}1 \mathrm{~h} \text { after } \\
\text { resuscitation (or } \\
\text { catheterization) }\end{array}$ & $136.78 \pm 4.68$ & $96.48 \pm 4.93 \Delta a$ & $113.03 \pm 4.18 \Delta \mathrm{ab}$ & $101.13 \pm 5.45 \Delta \mathrm{ac}$ & 111.329 & 0.000 \\
\hline & $\begin{array}{l}2 \mathrm{~h} \text { after } \\
\text { resuscitation (or } \\
\text { catheterization) }\end{array}$ & $136.29 \pm 5.15$ & $101.24 \pm 5.34 \Delta a$ & $114.23 \pm 4.78 \Delta \mathrm{ab}$ & $102.29 \pm 5.87 \Delta \mathrm{ac}$ & 75.485 & 0.000 \\
\hline & $\begin{array}{l}3 \mathrm{~h} \text { after } \\
\text { resuscitation (or } \\
\text { catheterization) }\end{array}$ & $138.88 \pm 3.98$ & $105.02 \pm 6.09 \Delta a$ & $117.28 \pm 5.13 \Delta a b$ & $103.86 \pm 5.56 \Delta \mathrm{ac}$ & 76.733 & 0.000 \\
\hline & $\begin{array}{l}4 \mathrm{~h} \text { after } \\
\text { resuscitation (or } \\
\text { catheterization) }\end{array}$ & $139.02 \pm 4.82$ & $105.40 \pm 5.71 \Delta a$ & $119.17 \pm 4.80 \Delta a b$ & $105.46 \pm 5.05 \Delta \mathrm{ac}$ & 77.379 & 0.000 \\
\hline
\end{tabular}

Remarks: Each rat underwent monitoring of blood pressure using femoral artery at different time points, including catheterization at $0,0.5,1,2,3$, and $4 \mathrm{~h}$ after resuscitation (or catheterization). Intra-group comparison: $\mathbf{\Delta}: \mathrm{P}<0.05$ compared with baseline; Intergroup comparison: a: $\mathrm{P}<0.05$ compared with sham-operated group; $\mathrm{b}: \mathrm{P}<0.05$ compared with CPR group; : $\mathrm{P}<0.05$ compared with CORM-2 group.

Table 2 Serum concentrations of I-FABP and the Chiu's scores

\begin{tabular}{|c|c|c|c|c|c|c|}
\hline Parameter & Sham-operated group & CPR group & CORM-2 group & iCORM-2 group & F-value & P-value \\
\hline & $\nabla n=8 \nabla$ & $\bigotimes n=8 \rrbracket$ & $\nabla n=8 \otimes$ & $\bigotimes n=8 \rrbracket$ & & \\
\hline I-FABP (pg/ml) & $230.19 \pm 37.01$ & $585.64 \pm 119.84 a$ & $306.10 \pm 19.22 b$ & $414.12 \pm 36.13 a b c$ & 43.689 & 0.00 \\
\hline Chiu's Scores & $0.40 \pm 0.13$ & $4.59 \pm 0.34 a$ & $2.38 \pm 0.38 a b$ & $3.64 \pm 0.53 a b c$ & 187.588 & 0.00 \\
\hline
\end{tabular}

Remarks: CO decreased intestinal injury and serum I-FABP level in CA/CPR rats. Semi-quantitative intestinal injury was evaluated by two investigators through the Chiu scoring of mucosal injury. The blood samples were drawn from the portal vein at $4 \mathrm{~h}$ after resuscitation. The serum I-FABP level was measured by ELISA kits. CPR led to histopathological injury in ileum tissues and CORM-2 treatment decreased injury in ileum tissues. iCORM-2 had no significant effect on injury in ileum tissues in CPR rats (a: $P$ $<0.05$ compared with the sham-operated group); b: $\mathrm{P}<0.05$ compared with the CPR group; $\mathrm{c}$ : $<<0.05$ compared with the CORM-2 group).

\section{Figures}



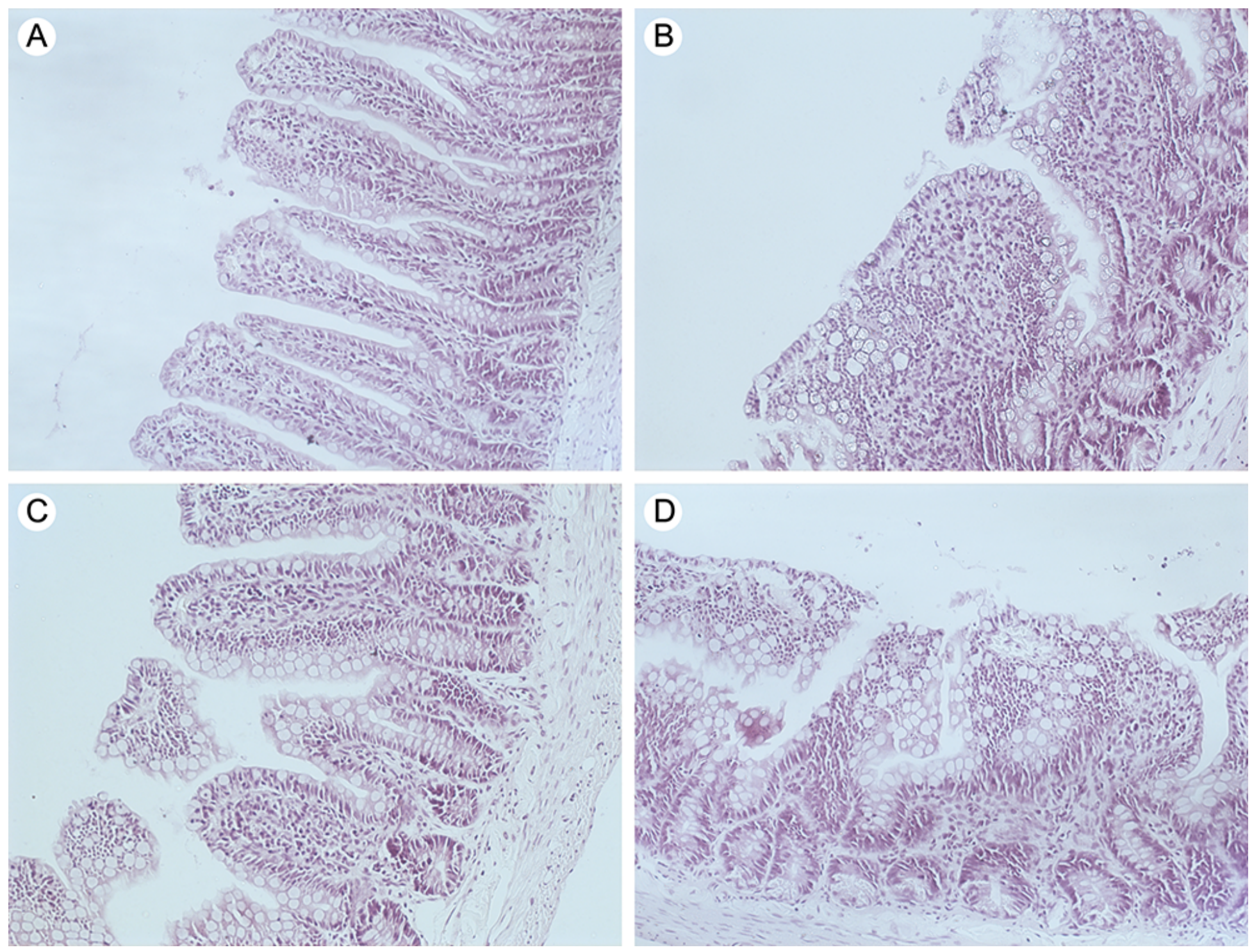

\section{Figure 1}

The histopathological morphology of rats in each group (magnification, 200x). When rats underwent CA-CPR, the small intestine was excised for hematoxylin-eosin staining. Typical images of H\&E staining of intestinal sections from sham-operated (A), CPR (B) rats or CPR rats undergoing CORM-2 (C) or iCORM-2 (D) treatment. Severe inflammation (ulceration, hemorrhage and epithelial loss) was found in CPR rats (B) and CPR rats treated with iCORM-2 (D). The changes were alleviated in CPR rats treated with CORM-2 (C). 

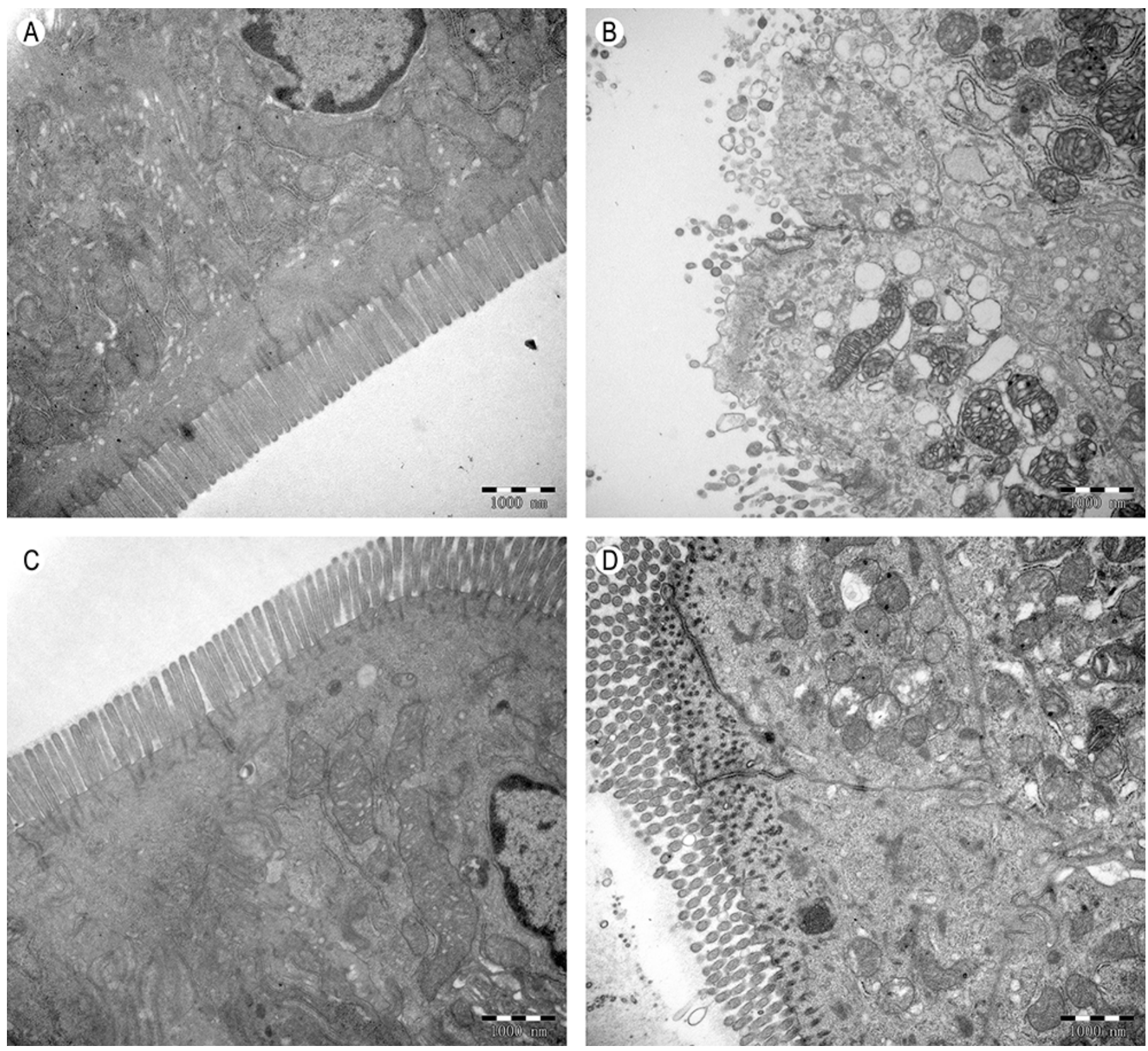

\section{Figure 2}

Ultrastructural changes in ileum of rats (magnification, 20000x). When rats underwent CA/CPR, the small intestine was excised and was analyzed by electron microscopy with lead-uranyl acetate. Typical images from CPR (B) and iCORM-2 (D) rats included widened intercellular space, swollen mitochondria, and absent microvilli. Ultrastructural changes were alleviated in the CORM-2 (C) group compared with those in the other groups. Sham-operated rats (A) showed a normal ultrastructure of the intestinal epithelium. 


\section{sham CPR CORM-2 iCORM-2}

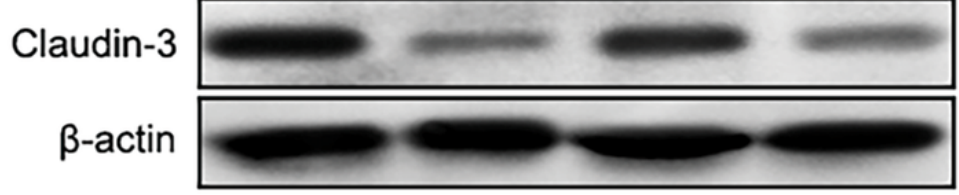

\section{$24 \mathrm{kDa}$}

\section{$42 \mathrm{kDa}$}
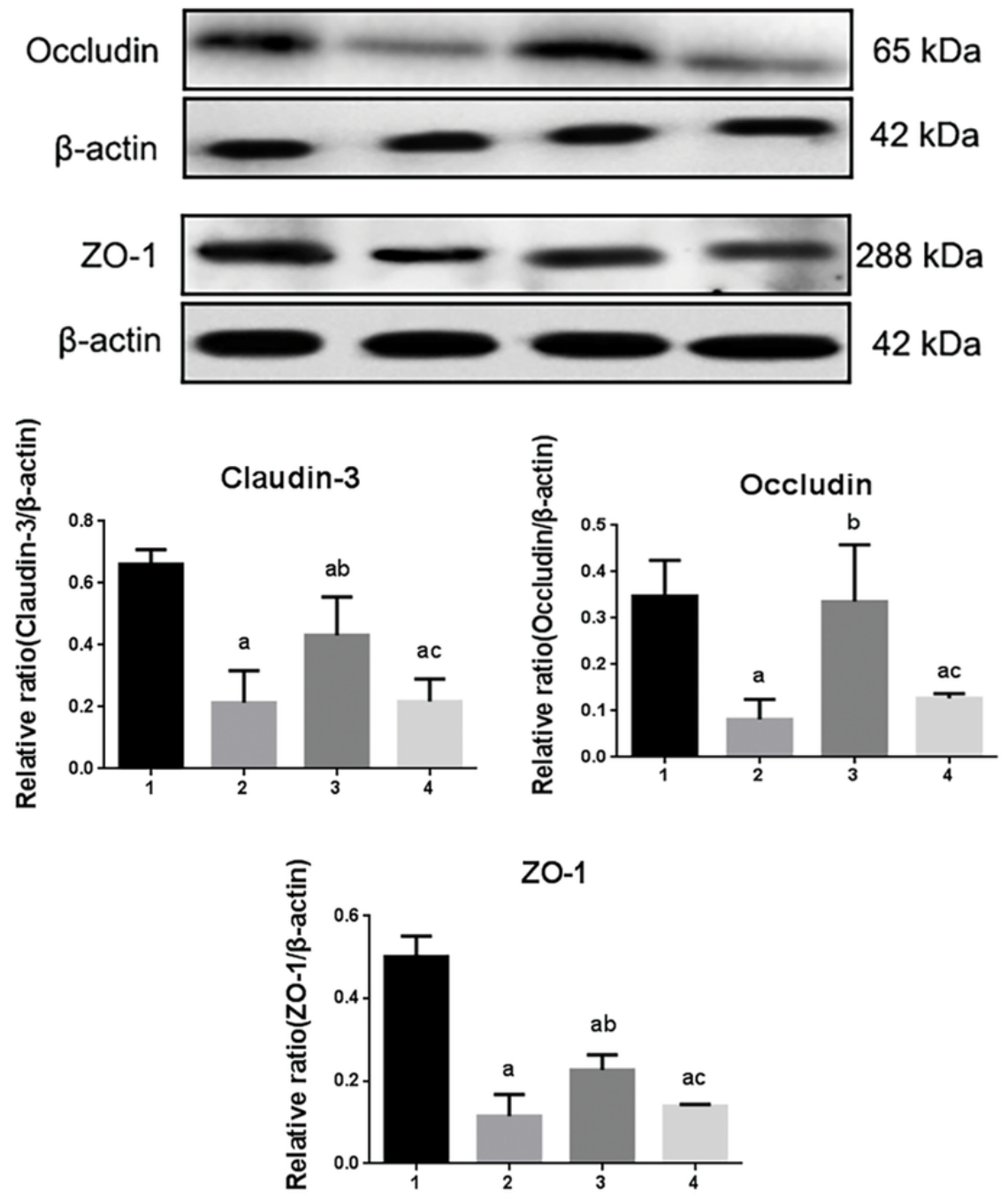

\section{Figure 3}

Relative expression levels of claudin-3, ZO-1, and occludin in the ileum tissue of rats. lleum samples were collected and homogenized. The tissue lysates were subjected to Western blotting using specific antibodies for claudin-3, ZO-1, and occludin with $\beta$-actin as an endogenous control. The band intensity was quantified using densitometry. The ratios of claudin-3/ $\beta$-actin, ZO$1 / \beta$-actin, and Occludin/ $\beta$-actin in different groups were presented as mean \pm SD. (1) sham-operated group; (2) CPR group; (3) CORM-2 group; (4) iCORM-2 group ( $\mathrm{n}=8$ for each group). a: $\mathrm{P}<0.05$ compared with sham-operated group; $\mathrm{b}: \mathrm{P}<0.05$ compared with CPR group; $\mathrm{C:} \mathrm{P}<0.05$ compared with CORM-2 group). 

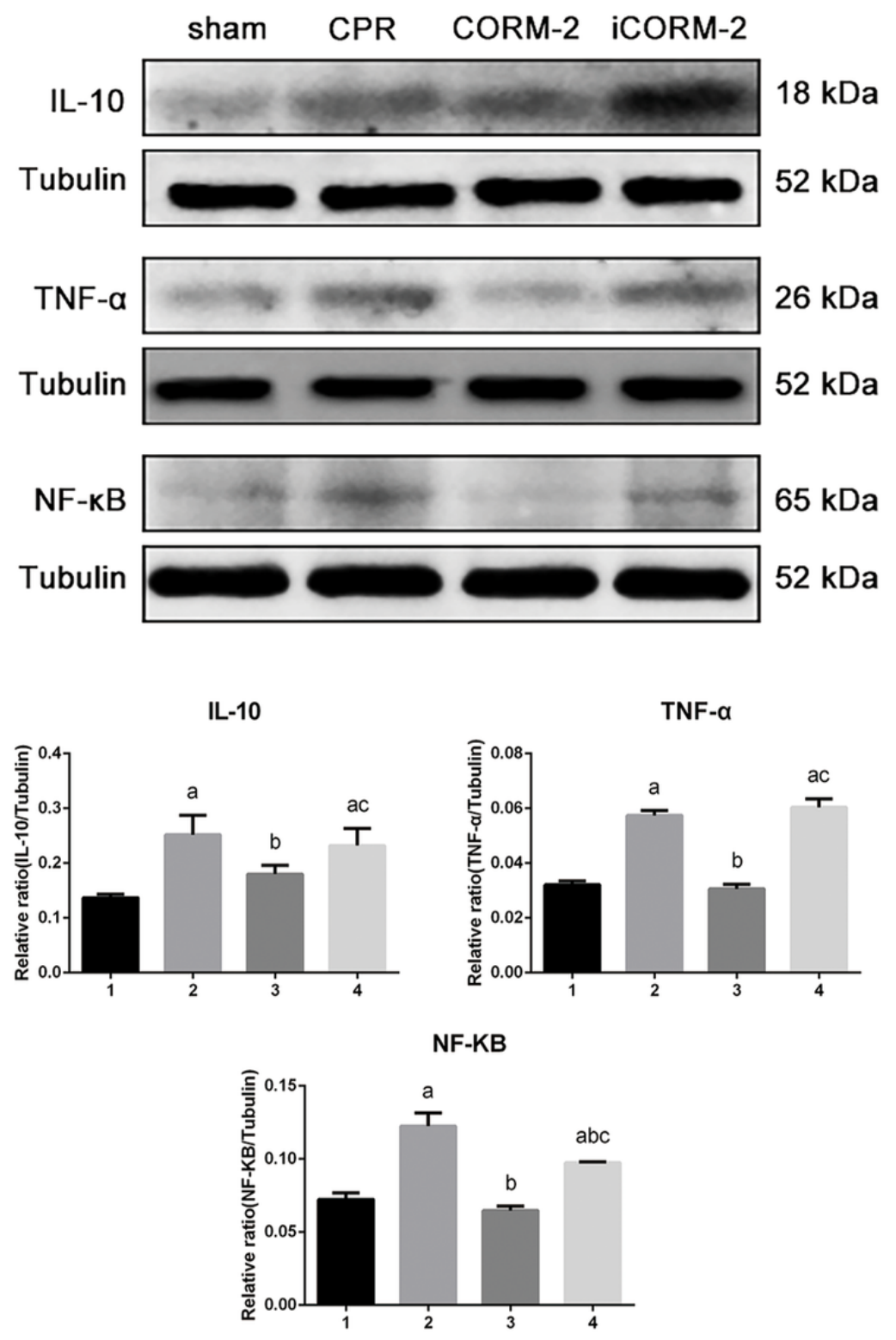

\section{Figure 4}

Relative expression levels of TNF-a, IL-10, and NF-KB p65 in ileum tissues of rats. The lysates of ileum in different groups of rats were subjected to Western blotting with anti-TNF-a, anti-IL-10, and anti-NF-KB p65 antibodies with Tubulin as an endogenous control. The ratios of TNF- $\alpha$ /Tubulin, IL-10/Tubulin, and NF-KB p65/Tubulin were calculated based on the densities of bands. (1) sham-operated group; (2) CPR group; (3) CORM-2 group; (4) iCORM-2 group ( $n=8$ for each group). a: $\mathrm{P}<0.05$ compared with the sham-operated group; b: $\mathrm{P}<0.05$ compared with CPR group; $\mathrm{C}: \mathrm{P}<0.05$ compared with CORM-2 group. 